\title{
Dosimetric analysis of the effects of the bladder volume on organs at risk (OAR) in high-dose-rate intracavitary brachytherapy in carcinoma cervix - an institutional study
}

\author{
Ashutosh Das Sharma, MD, Jyoti Poddar, MD, U Suryanarayan K, MD, Sonal Patel Shah, MD, \\ Ankita Parikh, MD, Vimesh Mehta, Med Phys, Tarun Kumar, Med Phys \\ Department of Radiotherapy, Gujarat Cancer and Research Institute, Ahmedabad, Gujarat, India
}

\begin{abstract}
Purpose: The purpose of this study was to establish a dosimetric correlation between the bladder volume and its effects on the dose received by the organs at risk (OARs) (urinary bladder, rectum, and sigmoid) during computed tomography (CT)-guided high-dose-rate (HDR) brachytherapy in carcinoma cervix, and to determine an optimum bladder volume to limit the dose to OARs.

Material and methods: Seventy-five intracavitary applications in patients of carcinoma cervix (stage IIB, IIIA, IIIB, IVA) treated with external beam radiotherapy with concurrent chemotherapy followed by CT-based HDR intracavitary brachytherapy (tandem and ovoid type) at our institute between July 2014 to January 2016 were studied. The bladder volume at the time of imaging was noted and was correlated with the radiation dose received by bladder, rectum, and sigmoid colon.

Results: Dose volume histogram (DVH) parameters of the bladder increases by elevating the volume of the bladder. Rectum dose does not follow a continuous increasing trend. It increases up to a bladder volume of $110 \mathrm{cc}$ and then starts decreasing. The highest rectal dose observed was in the bladder volume, range 70-110 cc. The minimum doses were recorded when the bladder volume was $>170 \mathrm{cc}$. Sigmoid colon DVH parameters follow a similar trend as that of the rectum.

Conclusions: A relationship exists between the volume of the OARs and the dose received by them. A bladder volume of about $70 \mathrm{~cm}^{3}$ or less proved better for achieving the prescribed dose limits of bladder, rectum, and sigmoid. The correlations between the bladder volume and the doses received by the OARs were not significant.
\end{abstract}

J Contemp Brachytherapy 2018; 10, 1: 26-31 DOl: https://doi.org/10.5114/jcb.2018.74136

Key words: image-guided brachytherapy, bladder volume, dosimetry, cervical carcinoma.

\section{Purpose}

Although, over decades, brachytherapy has improved the local control and overall survival in cervix carcinoma, the radiation-induced morbidity of the organs at risk (OARs) is unpredictable [1]. The radiation-induced bladder morbidity includes acute and chronic cystitis, hematuria, contracted bladder, and vesico-vaginal fistula [2]. The rectal injury includes recto-vaginal fistula and bleeding per rectum. A treatment can be deemed successful, if it provides maximal tumor coverage with minimal complications. Since the publication of International Commission on Radiation Units and Measurements (ICRU) report 38 in 1985, multiple studies have shown that the ICRU point dose may not represent the highest dose to the OARs, especially the bladder [3]. In 2005 and 2006, GEC-ESTRO (the Groupe Europeen de Curietherapie-European Society for Radiotherapy and Oncology) published recommendations on three-dimensional (3D) image-guided intracavitary brachytherapy, target definitions, and dose volume histogram (DVH) parameters, which has permitted better evaluation than ICRU point doses $[4,5]$. This has allowed a better understanding of the DVH effects on the OARs, and has showed that there is a linear correlation between the complications and the $D_{2 c c}$ received by the OARs. These recommendations emphasized the importance of geometric relationship between the brachytherapy applicator and the OARs to reduce the dose to these organs. Many studies have been

Address for correspondence: Jyoti Poddar, MD, Assist. Prof., Department of Radiotherapy, Gujarat Cancer Received: 27.11.2017 and Research Institute, Civil Hospital, Asarwa, 380016 Ahmedabad, India, phone: +91 8758270075, 
carried out so far, focusing on various methods to limit the dose to the OARs e.g., organ motion, changes in rectal and bladder volume, geometry of the applicator, use of rectal separator, vaginal packing, etc. $[3,6,7,8]$. Alteration of volume of the OARs has got an effect on organ dosimetry. Alteration of bladder volume, its shape and position, can alter the relative anatomy of uterus, sigmoid colon intestinal loops, and rectum. Various bladder filling protocols have been suggested but there is no clear consensus. It varies from empty bladder with indwelling Foley's catheter (ICRU 38 recommendations) to a known limited filling status (e.g., $50 \mathrm{cc}$ ) to a full bladder. In the ICRU 89 report, no recommendations have been put forward about the bladder volume status (full or empty bladder) for treatment of pelvic intracavitary brachytherapy (ICBT) [9]. Different aspects of this concept have been explored by multiple studies. Researchers have found that a full bladder might shift the sigmoid colon and intestinal loops away from the uterus, thereby having a sparing effect on sigmoid colon, intestinal loop, and on rectum [10]. A full bladder decreases the mean dose to the bladder itself, as it enlarges and shifts away from the target [7]. A second group of studies propose that, if brachytherapy is delivered with a full bladder, it increases the dose to the base of the bladder, as the base comes near to the target on distension [11]. Few researchers found that the bladder volume does not have any significant effect on the bladder dose, though it affects the rectal and sigmoid dose [12]. It is quite evident from all these studies that DVH of OARs and clinical target volumes (CTVs), recommended by GEC-ESTRO, are affected by bladder volume but none of these studies could find an optimum bladder volume to minimize the dose to the OARs [13]. This study aims at exploring the correlation between the volume of bladder and its effect on the bladder dose, during planning of brachytherapy. It was completed to formulate an institutional policy to be followed for all patients of 3D ICRT, which can be reproduced, and an optimum bladder volume can be calculated to decrease the dose to the OARs.

\section{Material and methods}

\section{Patients}

Seventy-five ICBT applications in patients of carcinoma cervix, treated at our institute between July 2014 and January 2016 were selected. The inclusion criteria were as follows: 1. Patients of carcinoma cervix of FIGO stage IIB to IVA (IIB, IIIA, IIIB, IVA); 2. Patients without any history of previous pelvic irradiation, pelvic surgery, or recurrent cervical cancer; 3 . A candidate of ICBT after external beam radiotherapy (EBRT), i.e. patient not requiring interstitial needle implant for bulky disease.

All patients were treated with external beam radiotherapy and concurrent chemotherapy. It was followed by 3D ICBT by HDR brachytherapy remote after-loading system $\left({ }^{192} \mathrm{Ir}\right)$. All the patients had received HDR brachytherapy with computed tomography/magnetic resonance imaging (CT/MRI) compatible tandem and ovoids type applicator.

\section{Methods}

All patients had received EBRT with concomitant cisplatin on a $6 \mathrm{MV}$ linear accelerator. One week after conclusion of the external radiation, the patients were admitted. They were administered laxative suppository 12 hours prior to the brachytherapy procedure, and the patients were asked to urinate just before the beginning of the brachytherapy procedure.

Patients were given spinal anesthesia and were examined in lithotomy position to document the extent of the disease. After sterile prepping and draping of the patient, a Foley's catheter was inserted with $1 \mathrm{cc}$ dye and $6 \mathrm{cc}$ normal saline. The uterus was sounded (with ultrasound guidance as and when required) and serial dilation of the cervical os was done, which was followed by insertion of the intrauterine tandem. Depending on the disease extent and vaginal distensibility, ovoids were placed. All the patients were treated with tandem and ovoids type of applicator. Different tandem lengths, i.e. $4 \mathrm{~cm}, 5 \mathrm{~cm}$, and $6 \mathrm{~cm}$ were used depending upon the length of the uterine cavity.

\section{Imaging and contouring}

All patients underwent CT simulation with $2.5 \mathrm{~mm}$ axial image slices. All patients had Foley's catheter. The catheter was clamped at the time of CT simulation and the volume of the bladder was recorded as obtained from the CT scan. The patients were classified into four groups, depending on the bladder volume. Group A: up to $70 \mathrm{cc}$, group B: 70-110 cc, group C: 110-170 cc, group D: > 170 cc. The images were obtained with the patient in treatment position (normal anatomical position). The CT images were transferred to the treatment planning system. The external contours of the sigmoid, rectum, and bladder were drawn. The high-risk clinical target volume (HR-CTV) and the intermediate risk clinical target volume (IR-CTV) were delineated by a radiation oncologist as per the GECESTRO GYN working group recommendations and using the available CT images as guide for delineation (at the time of diagnosis and also before the brachytherapy).

The prescription for HDR brachytherapy was 7.5 Gy/ fraction to HR-CTV. Equivalent dose $\left(\mathrm{EQD}_{2}\right)$ was calculated for HDR treatments using the linear quadratic model normalized to $2 \mathrm{~Gy} /$ fraction, using $\alpha / \beta$ of 3 for OARs and 10 for tumor [14].

\section{Treatment planning}

The image-guided brachytherapy plan was generated on the Oncentra treatment planning system (version 4.3 ), and the plan was optimized using geometrical and manual optimization. The post-implant dose-volume histograms (DVHs) of the target, rectum, bladder, and sigmoid (organs at risk) were generated. Dose to HR-CTV was noted and the $\mathrm{D}_{2 \mathrm{cc}}, \mathrm{D}_{1 \mathrm{cc},}$ and $\mathrm{D}_{0.1 \mathrm{cc}}$ of rectum, bladder, and sigmoid were noted from the DVHs.

\section{Statistical analysis}

Data analysis was performed using Pearson correlation and one-way ANOVA. A $p$ value of less than 0.05 was 
Table 1. Classification of four patients' groups according to the bladder volume

\begin{tabular}{lccc} 
Group & $\begin{array}{c}\text { No. of } \\
\text { applications } \\
\text { (total } n=75)\end{array}$ & $\begin{array}{c}\text { Bladder volume } \\
\text { in cc }\end{array}$ & $\begin{array}{c}\text { Mean bladder } \\
\text { volume in cc }\end{array}$ \\
\hline A & 37 & $0-70$ & 44 \\
\hline B & 20 & $70-110$ & 89 \\
\hline C & 09 & $110-170$ & 152 \\
\hline D & 09 & $>170$ & 188
\end{tabular}

considered statistically significant using SPSS (SPSS Statistics for Windows, version 17.0 Chicago: SPSS Inc. USA).

\section{Results}

\section{Classification}

The patients were classified into four groups A, B, C, and $\mathrm{D}$, as described above. The rationale of classifying the patients into subgroups was to correlate how the bladder volume influences the dose to the OAR. As it is a retrospective study, there is a skew towards patients having lower bladder volume. The classification of patients into four groups and the number of patients in each group is presented in Table 1.

\section{Data analysis}

The disease status i.e. HR-CTV volume was considered to be a confounding factor, and the correlation of the confounder was done with linear regression model. The linear regression and correlation analysis of the HRCTV with bladder volume was 0.2 , which shows the correla- tion, is weak and HR-CTV does not influence the bladder volume as a confounder.

\section{Effect of bladder volume on dose to the bladder}

The detailed dose patterns of bladder doses $\mathrm{D}_{2 \mathrm{cc}}, \mathrm{D}_{1 \mathrm{cc} \prime}$ and $\mathrm{D}_{0.1 \mathrm{cc}}$ for bladder were noted and tabulated in the respective group (Table 2). The mean, standard deviation, and variance for each group was calculated. From the above Table, it was calculated that when bladder volume increased from $70 \mathrm{cc}$ to $110 \mathrm{cc}$ (group A and B), the percentage increase in the mean $\mathrm{D}_{2 \mathrm{cc}}$ was $5.19 \%$. Similarly, when the bladder volume increased $>170 \mathrm{cc}$ (group A and $\mathrm{D})$, the mean $\mathrm{D}_{2 \mathrm{cc}}$ increased by $7 \%$.

When bladder volume increased from $<70 \mathrm{cc}$ to $>170 \mathrm{cc}$, the $D_{2 c c}$ dose increased by $19 \%$. The $p$ values were not found to be significant. Similar calculations were done for the rectal dose and the dose received by the sigmoid colon in each group, which is presented in Tables 3 and 4, respectively. The results obtained are depicted in Figure 1.

\section{Effect of bladder volume on dose to rectum}

The dose patterns of rectal doses $\mathrm{D}_{2 \mathrm{cc}}, \mathrm{D}_{1 \mathrm{cc}{ }^{\prime}}$ and $\mathrm{D}_{0.1 \mathrm{cc}}$ for rectum were noted and tabulated in the respective groups (Table 3). The rectum dose increased, as the bladder volume increased up to the volume of 110 cc. For higher bladder volumes, the rectum dose showed a decreasing trend. From the Table it is evident that the dose to the rectum was maximum, when the bladder volume was about $70-110 \mathrm{cc}$. In this range, the $\mathrm{D}_{2 \mathrm{cc}}, \mathrm{D}_{1 \mathrm{cc},}$ and $\mathrm{D}_{0.1 \mathrm{cc}}$ of the rectum became about $71.4 \%, 79.6 \%$, and $99 \%$ of the planning aim dose, respectively. All the reported doses are a mean dose. However, these rectum DVH parameters $\left(D_{2 c c}, D_{1 c c}\right.$ and $\left.D_{0.1 c c}\right)$ were $68 \%, 77 \%$, and $97 \%$, respectively, when the bladder volume was less than $70 \mathrm{cc}$.

Table 2. Bladder dose distribution pattern in different groups

\begin{tabular}{lcccc} 
Group & $\begin{array}{c}\text { Mean bladder volume in cc } \\
\text { (no of applications) }\end{array}$ & $\begin{array}{c}\mathrm{D}_{2 c c} \\
\text { Mean } \pm \text { SD }\end{array}$ & $\begin{array}{c}\mathrm{D}_{1 \mathrm{cc}} \\
\text { Mean } \pm \text { SD }\end{array}$ & $\begin{array}{c}\mathrm{D}_{0.1 \mathrm{cc}} \\
\text { Mean } \pm \text { SD }\end{array}$ \\
\hline $\mathrm{A}$ & $44(37)$ & $8.28 \pm 2.59$ & $9.30 \pm 3.09$ & $11.91 \pm 4.54$ \\
\hline $\mathrm{B}$ & $89(20)$ & $8.71 \pm 2.35$ & $9.56 \pm 2.63$ & $11.91 \pm 3.83$ \\
\hline $\mathrm{C}$ & $09(152)$ & $8.74 \pm 1.42$ & $10.21 \pm 2.48$ & $13.55 \pm 4.77$ \\
\hline $\mathrm{D}$ & $09(188)$ & $8.96 \pm 1.52$ & $9.91 \pm 1.70$ & $12.42 \pm 2.27$ \\
\hline$P$ value & & 0.8167 & 0.8109 & 0.7462
\end{tabular}

$D_{2 c c}$-the minimum doses to the highest irradiated $2 \mathrm{cc}$ area, $D_{1 c c}$ - the minimum doses to the highest irradiated $1 c c$ area, $D_{0.1 c c}-$ the minimum dose to the highest irradiated 0.1 cc area

Table 3. Rectum dose distribution pattern in different groups

\begin{tabular}{lcccc} 
Group & No. of applications & $\begin{array}{c}\mathrm{D}_{2 c c} \\
\text { Mean } \pm \text { SD }\end{array}$ & $\begin{array}{c}\mathrm{D}_{1 \mathrm{cc}} \\
\text { Mean } \pm \text { SD }\end{array}$ & $\begin{array}{c}\mathrm{D}_{0.1 \mathrm{cc}} \\
\text { Mean } \pm \text { SD }\end{array}$ \\
\hline $\mathrm{A}$ & 37 & $5.15 \pm 1.84$ & $5.83 \pm 2.01$ & $7.31 \pm 2.75$ \\
\hline $\mathrm{B}$ & 20 & $5.36 \pm 1.48$ & $5.97 \pm 1.48$ & $7.44 \pm 2.61$ \\
\hline $\mathrm{C}$ & 09 & $4.95 \pm 1.52$ & $5.59 \pm 2.10$ & $6.39 \pm 2.88$ \\
\hline $\mathrm{D}$ & 09 & $5.06 \pm 2.32$ & $5.65 \pm 2.78$ & $7.11 \pm 4.03$ \\
\hline$p$ value & & 0.9390 & 0.9603 & 0.8256
\end{tabular}

$D_{2 c c}$-the minimum doses to the highest irradiated $2 c c$ area, $D_{1 c c}$ - the minimum doses to the highest irradiated $1 c c$ area, $D_{0.1 c c}-$ the minimum dose to the highest irradiated $0.1 \mathrm{cc}$ area 
Table 4. Sigmoid colon dose distribution pattern in different groups

\begin{tabular}{lcccc}
$\begin{array}{l}\text { Group } \\
(n)\end{array}$ & Volume of bladder in cc & $\begin{array}{c}\mathrm{D}_{2 c c} \\
\text { Mean } \pm \text { SD }\end{array}$ & $\begin{array}{c}\mathrm{D}_{1 c c} \\
\text { Mean } \pm \text { SD }\end{array}$ & $\begin{array}{c}\mathrm{D}_{0.1 c c} \\
\text { Mean } \pm \text { SD }\end{array}$ \\
\hline $\mathrm{A}(37)$ & $<70$ & $3.53 \pm 1.69$ & $3.97 \pm 1.98$ & $5.08 \pm 2.58$ \\
\hline $\mathrm{B}(20)$ & $70-110$ & $4.58 \pm 1.86$ & $5.13 \pm 2.00$ & $6.38 \pm 2.42$ \\
\hline $\mathrm{C}(09)$ & $110-170$ & $4.44 \pm 2.34$ & $4.93 \pm 2.76$ & $6.76 \pm 3.37$ \\
\hline $\mathrm{D}(09)$ & $>170$ & $3.42 \pm 0.97$ & $3.87 \pm 1.20$ & $4.99 \pm 1.58$ \\
\hline$p$ value & & 0.1152 & 0.1474 & 0.1329
\end{tabular}

$D_{2 c c}$ - the minimum doses to the highest irradiated $2 c c$ area, $D_{1 c c}$ - the minimum doses to the highest irradiated $1 c c$ area, $D_{0.1 c c}-$ the minimum dose to the highest irradiated 0.1 cc area

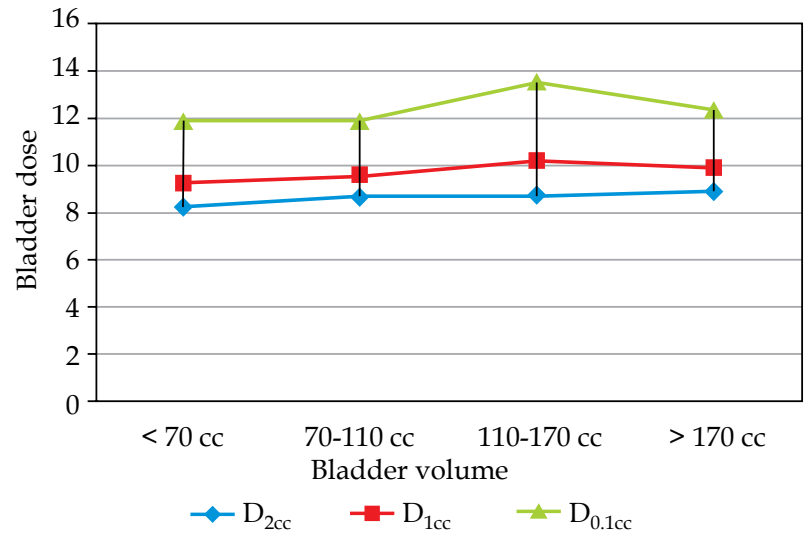

$D_{2 c c}$ - the minimum doses to the highest irradiated 2 cc area,

$D_{1 c c}$ - the minimum doses to the highest irradiated $1 \mathrm{cc}$ area,

$D_{0.1 c c}$ - the minimum dose to the highest irradiated $0.1 \mathrm{cc}$ area

Fig. 1. The variation of bladder dose with bladder volume

These parameters were $67 \%, 75 \%$, and $94 \%$, respectively, when the bladder volume was $>170 \mathrm{cc}$. The $\mathrm{p}$ values were not found to be significant. The results obtained are shown in Figure 2.

\section{Effect of bladder volume on dose of sigmoid}

The dose pattern of sigmoid $\mathrm{D}_{2 \mathrm{cc}}, \mathrm{D}_{1 \mathrm{cc}}$ and $\mathrm{D}_{0.1 \mathrm{cc}}$ for sigmoid colon were noted and showed in Table 4.

From Table 4, it is evident that the dose pattern obtained in sigmoid colon was similar to that of the rectum, i.e., the sigmoid dose increased with the increase in bladder volume up to $110 \mathrm{cc}$ and then it showed gradual fall. The minimum dose to the sigmoid colon was obtained when the bladder volume was $>170 \mathrm{cc}$. In this range, the $\mathrm{D}_{2 \mathrm{cc}}, \mathrm{D}_{1 \mathrm{cc}}$ and $\mathrm{D}_{0.1 \mathrm{cc}}$ were approximately $45.6 \%, 51.6 \%$, and $66 \%$ of the planning aim dose, respectively. The dose was maximum, when the bladder volume was in the range of 70-110 cc. It was $61 \%, 68 \%$, and $85 \%$ for $\mathrm{D}_{2 \mathrm{cc}}, \mathrm{D}_{1 \mathrm{cc}}$ and $\mathrm{D}_{0.1 \mathrm{cc}}$ of the planning aim dose, respectively. The $D_{2 c c}$ dose was about $15 \%$ less, when the bladder volume was $>170 \mathrm{cc}$, as compared to when it was 70-110 cc. The $p$ values were not found to be significant. The results obtained are shown graphically in Figure 3. According to GEC-ESTRO recommendations, $\mathrm{D}_{2 \mathrm{cc}}$ correlates with the late toxicity of the bladder, rectum, and sigmoid. $\mathrm{D}_{1 \mathrm{cc}}$ and $\mathrm{D}_{0.1 \mathrm{cc}}$ are mentioned for recording and documentation of dose.

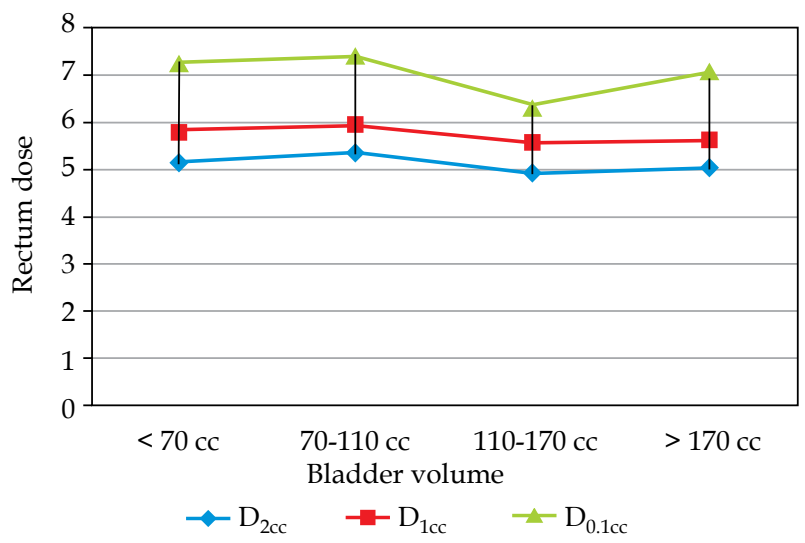

$D_{2 c c}$-the minimum doses to the highest irradiated $2 \mathrm{cc}$ area,

$D_{1 c c}$ - the minimum doses to the highest irradiated $1 \mathrm{cc}$ area,

$D_{0.1 c c}$ the minimum dose to the highest irradiated $0.1 \mathrm{cc}$ area

Fig. 2. The variation of rectal dose with bladder volume

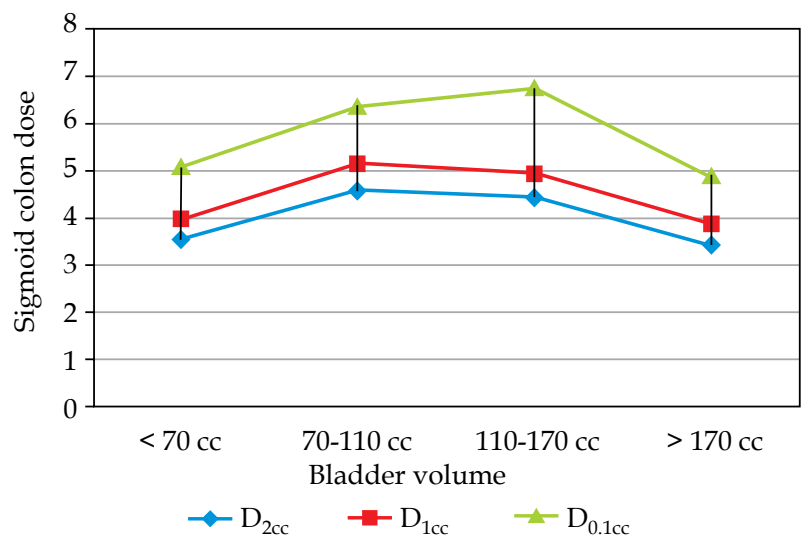

$D_{2 c c}$ - the minimum doses to the highest irradiated 2 cc area,

$D_{1 c c}$ - the minimum doses to the highest irradiated 1 cc area;

$D_{0.1 c c}$ - the minimum dose to the highest irradiated $0.1 \mathrm{cc}$ area

Fig. 3. The variation of sigmoid colon dose with change in bladder volume

\section{Discussion}

The effect of bladder volumes on DVH parameters of bladder, rectum, and sigmoid colon in HDR intracavitary brachytherapy of cervical cancer patients was assessed. The different parameters like bladder filling, rectal filling, sigmoid motion, variations in vaginal packing have significant impact on the dosimetry. Alteration of bladder filling status, shape, and position of the bladder alters the 
relative anatomy of the other pelvic organs. This study investigated the dosimetric impact of bladder filling status during HDR brachytherapy. Also, the optimum volume range of the bladder was searched, so that within that range, the OAR would receive an optimum radiation dose. This study was carried out with an idea of establishing an institutional guidance for the bladder filling during ICBT of carcinoma cervix at our facility. We included small volume dose values $(0.1 \mathrm{cc}, 1 \mathrm{cc}, 2 \mathrm{cc})$ following GEC-ESTRO recommendations, as these volumes are the key parameters to evaluate late toxicities. During analysis, emphasis was given on $\mathrm{D}_{2 \mathrm{cc}}$ as this is the most relevant and frequent parameter, which correlates with late OAR toxicity.

Image-based HDR brachytherapy in cervical cancer has evolved a great deal with promising results. For decades, Foley's catheterization and bladder evacuation was practiced, and was proven to be beneficial for lowdose-rate brachytherapy (LDR), where the treatment time was longer, and patients' comfort was taken into consideration. There is no common consensus about what should be done for HDR brachytherapy. Many studies have shown that doses to the ICRU bladder point do not correlate well with bladder complications, although this point is easily reproducible $[3,15,16]$. We investigated the bladder distension effect on doses to bladder, rectum, and the sigmoid colon, in 75 applications of ICBT in patients of carcinoma cervix. In our study, we found that even in open Foley's catheter, there was residual urine volume of 50-70 cc in the bladder. We wanted to determine an acceptable bladder volume. There have been multiple studies showing different results.

Yamashita etal.studied bladder filling with $50 \mathrm{cc}, 100 \mathrm{cc}$, $150 \mathrm{cc}$, and $200 \mathrm{cc}$ with sterile water, and compared the dosimetry to empty bladder and evaluated the position of hot spots on the bladder. The mean bladder $\mathrm{D}_{2 \mathrm{cc}}$ increased significantly by 3.5 Gy from an empty to a full bladder: $4.2 \mathrm{~Gy}$ to $7.7 \mathrm{~Gy}(84.5 \%), p=0.001$ [11].

Kim et al. showed that an increase in bladder volume resulted in a significant reduction in $\mathrm{D}_{2 \mathrm{cc}}$ of the bowel, at the expense of a growth in $\mathrm{D}_{2 \mathrm{cc}}$ bladder volume doses. They concluded that treatment with full bladder (median value, $367 \mathrm{~cm}^{3}$ ) is preferable to protect the bowel at the expense of the bladder [17].

In contrast to these observations, two other studies found different results. Sun et al. did an analysis of 20 patients of carcinoma cervix with empty and full bladder and found that the association between bladder volume and median bladder wall absorbed dose was significant [7]. Bladder distension reduced the median bladder absorbed dose by approximately $48 \%$ in this study. The authors concluded that higher bladder volumes would decrease acute and late radiation-related complications. However, there was no clinical outcome data of this study and the OARs toxicity correlates with $\mathrm{D}_{2 \mathrm{cc}}$ rather than the median absorbed dose.

Cengiz et al. analyzed 10 cervical cancer patients with full and empty bladder and found no significant difference in the dose distributions between the empty and the full bladder group [10]. However, they had concluded that the bladder fullness significantly reduced the dose to the rectum, sigmoid colon, and the small intestine, but the bladder dose maximum and $\mathrm{D}_{5 \mathrm{cc}}$ bladder was significantly increased despite lower doses to the rest of the bladder wall. Therefore, they proposed that the distended bladder pushes the small intestine and rectum away from the applicators, resulting in a relatively low-dose to these structures, although the differences were small and the clinical significance was unknown. Harmon et al. did a study and found that bladder distension during cervical brachytherapy significantly reduced dose in all DVH parameters for sigmoid and small intestine, with no change in bladder parameters. It reduces dose to organ at risk, but the correlation to toxicity requires further investigation [18].

Despite the conclusions drawn by Sun et al. and Cengiz et al., that a full bladder would decrease the dose to the rectum, we hypothesized that a fuller bladder would push the whole implant towards the rectum, closer to the rectal wall, and thereby increasing the maximum rectal dose. This contrasting idea is in conflict with the previous proposition by other studies mentioned, and warrants a radiological evidence of how the gradually distending bladder changes the relative anatomy of pelvic organs in an implanted patient. Our results for the evaluation of bladder dose variation by increasing the bladder volumes are in close consistency with previous studies.

In our study, it was observed that when the bladder volume increased from $70 \mathrm{cc}$ to up to $>170 \mathrm{cc}$, the $\mathrm{D}_{2 \mathrm{cc}}$ of bladder increased by about $19 \%$ of the prescribed target dose. However, the dose to the bladder cannot be extrapolated for empty bladder. When bladder is completely empty, the anterior and posterior walls would oppose, thus increasing the total volume of bladder being irradiated. This effect could not be elicited in DVH analysis. Therefore, there is no evidence of treating the patients with empty bladder at this point of time.

In accordance with the results obtained for the rectum, it can be concluded that the bladder volume does affect the rectal doses. Increasing the bladder volume up to $110 \mathrm{cc}$ would push the applicator posterior towards the rectum, thus increasing the rectal dose. Beyond the bladder volume of $110 \mathrm{cc}$, this effect on rectal dose seems to be negligible. This statement holds good and is in accordance with various previous studies [8].

The dose to the sigmoid colon can be decreased by bladder filling up to $70 \mathrm{cc}$. For higher bladder volumes (> $110 \mathrm{cc}$ ), the effect turns opposite again, as in the case of the rectum. This can be explained, perhaps by taking into account the upward shift in the bladder position, when volume is higher, in turn shifting the sigmoid away from the implant. Another important point worth discussing here is the volume of the sigmoid and rectum itself, which affects the maximum doses received by them. Therefore, DVH parameters of rectum and sigmoid are an interplay between the rectal and sigmoid volume along with the bladder volume.

The sigmoid dose showed a negative correlation with bladder volume, which may be due to its relatively mobile nature, as compared to the more fixed anatomy of the bladder and rectum. Furthermore, the probable reason of 
obtaining both positive and negative correlation between bladder volume and sigmoid dose would be the partially fixed and incompletely mobile nature of sigmoid.

Considering the true pelvis, which is the site of interest, is a fixed enclosed space. It appears logical that 200-500 cc of extra volume in the form of urine in the bladder would decrease the available room in the true pelvis, and because of the unique, rapidly falling dose nature of brachytherapy, even a crowding of few mm of the OARs, may significantly increase their maximum dose.

ICRU 89 does not recommend a full or empty bladder for treatment of pelvic ICBT. According to the data available and findings of our study, it appears thoughtful to keep the bladder as empty as possible during all the phases of brachytherapy (implant, planning, and treatment delivery) to reduce the dose to the bladder and rectum. The limitations of the study are the length of the tandem that has not been taken into account, which can influence the dose received by the OAR. A study showed that the dose to bladder, rectum, and sigmoid is higher when longer tandems were used, as compared to the shorter ones [1]. Also, the volume of the rectum and the sigmoid have not been taken into consideration.

\section{Conclusions}

There is an optimum volume range for the bladder, rectum, and sigmoid, which affects the received dose during intracavitary brachytherapy. Though the $p$ values were not found to be significant in this study, the important observations and conclusion of this study are as follows: 1 . DVH parameters of the bladder increase by elevating the volume of the bladder; 2 . Rectum dose does not follow a continuous growing trend. It increases up to a bladder volume of $110 \mathrm{cc}$ and then again starts decreasing. The highest rectal doses were observed in the bladder volume, range 70-110 cc. The minimum doses were recorded when the bladder volume was $>170 \mathrm{cc}$; 3. Sigmoid colon DVH parameters follow a similar trend as that of the rectum. The highest doses were recorded when the bladder volume was 70-110 cc, and minimum when the bladder volume was $>170 \mathrm{cc}$.

To conclude, in HDR intracavitary brachytherapy in carcinoma cervix, a relationship exists between the volume of the OARs and the dose received by them. A bladder volume of about $70 \mathrm{~cm}^{3}$ or less proved better for achieving the prescribed dose limits of bladder, rectum, and sigmoid.

\section{Disclosure}

Authors report no conflict of interest.

\section{References}

1. Siavashpour Z, Aghamiri MR, Jaberi R et al. Optimum organ volume ranges for organs at risk dose in cervical cancer intracavitary brachytherapy. J Contemp Brachytherapy 2016; 8: 135-142.

2. Gellrich J, Hackenberg OW, Oehlschlager S et al. Manifestation, latency and management of late urological complications after curative radiotherapy for cervical carcinoma. Onkologie 2003; 26: 334-340.
3. Fellner C, Potter R, Knocke TH et al. Comparison of radiography and computed tomography-based treatment planning in cervix cancer in brachytherapy with specific attention to some quality assurance aspects. Radiother Oncol 2001; 58: 5362.

4. Haie-Meder C, Pötter R, Van Limbergen E et al. Recommendations from Gynaecological (GYN) GECESTRO Working Group (I): concepts and terms in 3D image-based 3D treatment planning in cervix cancer brachytherapy with emphasis on MRI assessment of GTV and CTV. Radiother Oncol 2005; 74: 235-245.

5. Pötter R, Haie-Meder C, Van Limbergen E. Recommendations from gynaecological (GYN) GEC ESTRO working group (II): concepts and terms in 3D image-based treatment planning in cervix cancer brachytherapy-3D image-based anatomy, radiation physics, radiobiology. Radiother Oncol 2006; 78: 67-77.

6. Patra NB, Manir KS, Basu S. Effect of bladder distension on dosimetry of organs at risk in computer tomography-based planning of high dose rate intracavitary brachytherapy for cervical cancer. J Contemp Brachytherapy 2013; 5: 3-9.

7. Sun LM, Huang HY, Huang EY. A prospective study to assess the bladder distension effects on dosimetry in intracavitary brachytherapy of cervical cancer via computer tomography assisted techniques. Radiother Oncol 2005; 77: 77-82.

8. Adli M, Garipagaoglu M, Kocak Z. Effect of bladder distension on bladder base dose in gynaecological intracavitary high dose rate brachytherapy. Br J Radiol 2009; 82: 243-248.

9. ICRU Report 89, Prescribing, Recording, and Reporting Brachytherapy for Cancer of the Cervix. www.icru.org

10. Cengiz M, Gurdalli S, Selek U. Effect of bladder distension on dose distribution of intracavitary brachytherapy for cervical cancer. Three-dimensional computed tomography plan evaluation. Int I Radiat Oncol Biol Phys 2008; 70: 464-468.

11. Yashamita H, Nakagawa K, Okuma K. Correlation between bladder volume and irradiated dose of small bowel in CTbased planning of Intracavitary Brachytherapy for Cervical Cancer. Jpn J Clin Oncol 2012; 42: 302-308.

12. Han K, Milosevic M, Fyles A et al. Trends in the utilization of brachytherapy in cervical cancer in the United States. Int J Radiat Oncol Biol Phys 2013; 87: 111-119.

13. Stewart AJ, Cormack RA, Lee $\mathrm{H}$ et al. Prospective clinical trial of bladder filling and three-dimensional dosimetry in high-dose-rate vaginal cuff brachytherapy. Int J Radiat Oncol Biol Phys 2008; 72: 843-848.

14. Kirisits $C$. The EQD2 concept for practical reporting for cervix brachytherapy. http://www.aoic.net/elekta/elk1402archive/D201Kirisits.pdf

15. Orton CG, Wolf-Rosenblum S. Dose dependence of complication rates in cervix cancer radiotherapy. Int J Radiat Oncol Biol Phys 1986; 12: 37-44.

16. Stryker JA, Bartholomew M, Velkley DE. Bladder and rectal complications following radiotherapy for cervix cancer. Gynaecol Oncol 1988; 29: 1-11.

17. Kim RY, Shen S, Lin HY. Effects of bladder distension on organs at risk in 3D image-based planning of intracavitary brachytherapy for cervical cancer. Int J Radiat Oncol Biol Phys 2010; 76: 485-488.

18. Harmon G, Chinsky B, Surucu M et al. Bladder distension improves the dosimetry of organs at risk during intracavitary cervical high-dose-rate brachytherapy. Brachytherapy 2016; 15: 30-34. 